\section{Comparison of visual field progression between temporally tilted disc and nontilted disc, in patients with normal tension glaucoma}

Y-J Choy' ${ }^{1}$ Y Kwun' ${ }^{2}$, JC Han ${ }^{2}$ and C Kee
'Department of Ophthalmology, Eul-ji University Hospital, Daejeon, Korea

${ }^{2}$ Department of Ophthalmology, Samsung Medical Center, Sungkyunkwan University School of Medicine, Seoul Korea

Correspondence: C Kee, Department of Ophthalmology, Samsung Medical Center, Sungkyunkwan University School of Medicine, 81 Irwon-ro, Gangnam-gu, Seoul 135-710, Korea Tel: +82 234103548 ; Fax: +82 234100074 . E-mail: ckee@skku.edu

Received: 27 March 2014 Accepted in revised form: 1 January 2015 Published online: 27 February 2015

\section{Abstract}

Purpose To investigate the long-term visual field (VF) progression of temporally tilted disc and nontilted disc in normal tension glaucoma (NTG).

Methods Retrospective, observational case series. Forty-seven patients with temporally tilted disc (47 eyes), 44 patients with nontilted disc in NTG (44 eyes) patients, who were examined by at least $5 \mathrm{VF}$ tests, and were followed-up over a 5-year period, at the Department of Ophthalmology of the Samsung Medical Center, from May 1998 to 2013. VF progression was defined by modified Anderson-Hodapp criteria, and Glaucoma Progression Analysis (GPA). Multivariate analysis was used to identify the risk factors for VF progression in the temporally tilted disc. Results According to the Anderson-Hodapp criteria, progression rates of the temporally tilted disc and nontilted disc at $\mathbf{6 0}$ months were $19 \%$ and $72 \%$, respectively $(P<0.0001)$. According to GPA, they were $25 \%$ and $53 \%$, respectively $(P<0.0001)$. Twenty of 47 patients in the temporally tilted disc did not show progression. Among them, the more tilted disc showed the more VF defects. The hazard ratio of retinal nerve fiber layer (RNFL) defect type was 3.08 (95\% CI, 1.17-8.14; $P=0.02)$. The simultaneous superior and inferior RNFL defect type was the most common in progressors in the temporally tilted disc $(P=0.04)$.

Conclusion Through long-term follow-up, the cumulative survival rate of temporally tilted disc was higher than that of nontilted disc. Caution is required in the treatment of the temporally tilted disc. New treatment policy for the temporally tilted disc may follow. Eye (2015) 29, 1308-1314; doi:10.1038/eye.2015.17; published online 27 February 2015
Introduction

Glaucoma is caused by a morphologic change of the lamina cribrosa (LC), such as backward bowing and disorganization of the LC. ${ }^{1,2}$ The LC is a multilayered sieve-like structure in the sclera, where retinal ganglion cell axons exit from the eye. ${ }^{3}$

Glaucoma is a multifactorial disease. Myopia is one of the risk factors for the development and the progression of glaucoma. The Beaver Dam study reported that the incidence rate of glaucoma in myopic patients was 1.6 times higher than that in those with emmetropia, ${ }^{4,5}$ and the Blue Mountains Eye Study reported that myopia patients have a two-fold to three-fold increased risk of glaucoma compared with that of nonmyopic subjects. ${ }^{6}$

Myopic tilted disc may bring out the transformation of the LC and affect the retinal nerve fiber layer (RNFL), causing the RNFL defect. Blockage of the axonal transport within the LC has been proposed as a salient pathogenic mechanism for glaucoma. $3,7,8$ The myopic tilted disc and peripapillary atrophy may increase the intraocular pressure (IOP)related strain placed on certain axons. ${ }^{9}$

The tilted disc merely caused by myopic change might not have progression, even if RNFL defects exist. In our unpublished data on patients with inferiorly tilted disc syndrome, initial glaucoma-like visual field (VF) defect did not always show the characteristic progression that defines the disease over a 10 year period.

Our study compared the long-term VF progression between temporally tilted disc and nontilted disc in normal tension glaucoma (NTG). In addition, we analyzed the risk factors associated with VF progression of the 
temporally tilted disc in NTG and evaluated the difference of progression rate in progressors, between the temporally tilted disc and nontilted disc, in NTG.

\section{Methods}

In this retrospective study, medical charts of 960 NTG patients were reviewed, who had undergone $>5 \mathrm{VF}$ tests and over the 5-year follow-up period at the Department of Ophthalmology of Samsung Medical Center, from May 1998 to 2013. A diagnosis of NTG was made, when a patient with an IOP of $21 \mathrm{~mm} \mathrm{Hg}$ or less, without treatment, had findings of glaucomatous optic disc damage (such as diffuse or localized rim thinning, disc hemorrhage, a notch in the rim or a vertical cup-to-disc ratio higher than that of the other eye by $>0.2),{ }^{10,11}$ and corresponding glaucomatous VF defects; an open angle, observed by gonioscopic examination; and no underlying cause for the optic disc damage aside from glaucoma. ${ }^{5,12}$

If the both eyes of the same patient were eligible, one eye was selected randomly. The patients were divided into two groups: Group 1 consisted of temporally tilted disc and Group 2 of nontilted disc, in NTG patients.

The inclusion criteria were as follows: (1) temporally tilted disc with tilt axes within 30 degrees of the vertical meridian, to rule out inferiorly tilted disc; 'index of tilt (IT)' calculated as quotient of the minimum diameter of the disc divided by its maximum, $\leq 0.8 ;{ }^{13}$ and baseline spherical equivalent (SE) $<8$ diopters (Ds); ${ }^{14}$ and (2) for nontilted disc, 'IT' over $0.9,{ }^{13}$ and emmetropes who had SE between +0.75 D and - 0.75 D. ${ }^{15}$

Exclusion criteria were visual acuity worse than 6/12; any history of any ocular surgery, except successful cataract surgery; evidence of uveitis, corneal opacity or scarring; media opacity, such as significant cataract; retinopathies that would affect VF, such as diabetic mellitus and hypertension, or neurologic abnormalities; and optic neuropathies. The following variables were recorded: age, sex, follow-up period, number of VF tests, baseline SE, number of topical medications, baseline IOP, mean IOP, peak IOP, baseline mean RNFL thickness, and baseline mean central corneal thickness (CCT). The IOP measurements were performed using a Goldmann applanation tonometry and the baseline RNFL thickness was measured by Stratus OCT (Carl Zeiss Meditec Inc., Dubin, CA, USA). The baseline CCT was measured by ultrasound pachymetry (Ultrasonic Pachometer, Humphrey Instruments Inc., San Leandro, CA, USA).

\section{Visual field examination}

The patients underwent automated VF test with the 30-2 SITA standard strategy (Humphrey 740 Visual Field Analyzer, Carl Zeiss Meditec Inc.). The VF tests were performed at baseline, at 3 months after initial intervention and at each 6 month or 1 year follow-up examination.

Reliability criteria were established as fixation losses $<20 \%$, false positive rate $<33 \%$, and false negative rate $<33 \%$. Progression of the glaucomatous VF in these subjects was defined using two methods: (1) modified Anderson-Hodapp criteria, ${ }^{16,17}$ and (2) Glaucoma Progression Analysis (GPA, Carl Zeiss Meditec Inc.) software, which supplies both an event-based, and a trend-based progression analysis. The VF progression by the modified Anderson-Hodapp criteria was defined as three adjacent points that had a 5-dB loss or more from the initial level of loss on the total deviation plot; and at least one of those points should have a $10-\mathrm{dB}$ loss or more on two consecutive VF tests. When the VF progression was detected by the modified Anderson-Hodapp criteria, the next VF test was also screened to confirm the progression and to rule out fluctuation.

Given event-based analysis, the GPA is significant $(P<0.05)$ when the deterioration is evident on the pattern deviation probability maps at the same three or more points on two consecutive follow-up tests, and the GPA flags this as 'possible progression'. If significant deterioration is seen at the same three or more points in three consecutive follow-up tests, GPA flags this as 'likely progression'. When the VF defect does not belong to the above, the software flags this as 'no progression detected'. ${ }^{18}$ The 'possible progression' and 'likely progression' were considered as VF progression. As trend-based analysis, GPA software provides the slope of the change of the visual field index (VFI), with respect to time. The VFI is the aggregate percentage of visual function for a given field, at each point where the visual thresholds are estimated. It is the progression rate of the visual function of the eye, through a linear regression model, using VFI. ${ }^{18-20}$

Visual field global indices, which are baseline mean deviation (MD), baseline PSD, and baseline VFI from VF tests, were recorded.

\section{Data analysis}

Statistical analyses were conducted, using software named R 3.0.1, 2013 (a language and environment for statistical computing R Foundation for Statistical Computing, Vienna, Austria). Comparison of the temporally tilted disc and the nontilted disc were performed, using an independent sample two-tailed $t$-test and the Mann-Whitney $U$-test. Kaplan-Meier survival analysis was used to assess the cumulative incidence probabilities of VF progression between the two groups. The Mann-Whitney $U$-test and $\chi^{2}$-test were performed to compare progressors and non-progressors, in the 
temporally tilted disc in NTG patients. The multivariate multiple cox regression model was used to identify the risk factors for the VF progression in the temporally tilted disc; hazard ratios (HRs) and bias corrected 95\% confidence intervals (CIs) were reported. That is, a $P$-value of $<0.05$ was considered significant.

\section{Results}

\section{Univariate analysis}

Descriptive statistics (temporally tilted disc vs nontilted disc in NTG patients) Ninety-one NTG patients were enrolled in the present study. Group 1 (temporally tilted disc in NTG patients) included 47 eyes from 47 individuals, 30 men and 17 women, aged between 31 and 88 years (mean \pm SD,

$52 \pm 10$; Table 1). Group 2 (nontilted disc in NTG patients) included 44 eyes from 44 individuals, 19 men and 25 women, aged between 40 and 87 years (mean \pm SD, $69 \pm 9.1)$. The group 2 patients were significantly older than the group 1 patients $(P<0.001$, independent sample twotailed $t$-test). The $\mathrm{SE}$ of each group was $-4.8 \pm 1.4$ and $0 \pm 0.4 \mathrm{D}$, respectively, and differed significantly between the two groups $(P=<0.001$, independent sample twotailed $t$-test). Progression rates of the VFI by the GPA were $-0.9 \pm 0.9 \% /$ year in group 1 , and $-1.6 \pm 1.1 \%$ /year in group 2 . The progression rates of group 2 were significantly faster than those of group 1. Except for the age, SE, and progression rates of the VFI, there were no significant differences in any variables between the two groups.

Visual field progressor and non-progressor in both the two groups were defined, based on the modified Anderson-Hodapp criteria. We compared the progression rates of the VFI between VF progressors $(n=20)$ in group 1 and the VF progressors $(n=39)$ in group 2 . The progression rates of each group were $-1.3 \pm 0.9 \%$ /year and $-1.7 \pm 1.1 \%$ /year, and revealed no statistically significant difference between the two groups ( $P=0.1$, Mann-Whitney $U$-test). Also, we compared the progression rates of VFI between VF progressors $(n=8)$ over $6 \mathrm{D}$ in group 1 and VF progressors $(n=39)$ in group 2 . They were $-1.2 \pm 1.1 \%$ /year in group 1 and $-1.7 \pm 1.1 \%$ /year in group 2 , and the difference between the two groups was not statistically significant $(P=0.16$, Mann-Whitney $U$-test).

Survival analysis Comparisons of the probabilities of VF progression were analyzed by Kaplan-Meier survival analysis. The probabilities of VF progression and time to VF progression of group 1 and group 2, by the modified Anderson-Hodapp criteria and the GPA, are shown in Figure 1. By the modified Anderson-Hodapp criteria, the progression rates of the two groups at 60 months were $19 \%$ in group 1 and $72 \%$ in the group 2 (Figure 1a). The differences of the cumulative progression rates between the two groups were statistically significant $(P<0.0001$, $\chi^{2}$-test). By the GPA, the progression rates at 60 months after initial visit were $25 \%$ in group 1 and $53 \%$ in group 2, respectively (Figure $1 \mathrm{~b}$ ). The differences of the cumulative progression rates between the two groups were statistically significant $\left(P<0.0001, \chi^{2}\right.$-test $)$.

Descriptive statistics (progressors vs non-progressors in temporally tilted disc in NTG patients) Subgroup analysis between the VF progressors and non-progressors of the temporally tilted disc in NTG patients were performed. The number of VF progressors were 20 and the

Table 1 Comparison of the clinical demographics between the two groups $(n=90)$

\begin{tabular}{lccc}
\hline Characteristics & $\begin{array}{c}\text { Group 1: temporally tilted disc } \\
(\mathrm{n}=47) \text { mean } \pm S D\end{array}$ & $\begin{array}{c}\text { Group 2: nontilted disc } \\
(\mathrm{n}=44) \text { mean } \pm S D\end{array}$ & $\begin{array}{c}\text { P-value } \\
\text { Age (years) }\end{array}$ \\
Gender male/female & $52 \pm 10.0(31-88)$ & $69 \pm 9.1(40-87)$ & $<0.001^{\mathrm{a}}$ \\
Follow-up period (months) & $30 / 17$ & $19 / 25$ & $0.04^{\mathrm{b}}$ \\
Spherical equivalent (D) & $108.2 \pm 32.5$ & $112.4 \pm 43.6$ & $0.61^{\mathrm{a}}$ \\
Number of topical medications & $-4.9 \pm 1.2(-7$ to -3$)$ & $-0.1 \pm 0.5(-0.7$ to 0.7$)$ & $<0.001^{\mathrm{a}}$ \\
Baseline IOP (mm Hg) & $1.4 \pm 0.5(1-3)$ & $1.2 \pm 0.5(1-3)$ & $0.16^{\mathrm{a}}$ \\
Mean IOP (mm Hg) & $16.3 \pm 2.7(10-21)$ & $15.8 \pm 2.5(11-21)$ & $0.28^{\mathrm{a}}$ \\
Peak IOP (mm Hg) & $15.6 \pm 2.3(12-19)$ & $14.4 \pm 2.0(10.5-19.1)$ & $0.08^{\mathrm{a}}$ \\
Baseline mean RNFL thickness $(\mu \mathrm{m})$ & $18.4 \pm 2.5(14-21)$ & $17.6 \pm 2.3(13-21)$ & $0.10^{\mathrm{a}}$ \\
Baseline mean CCT ( $\mu$ m) & $79.0 \pm 14.6(48.7-106.1)$ & $79.4 \pm 13.9(46.5-113.6)$ & $0.88^{\mathrm{a}}$ \\
Number of visual field tests & $530.2 \pm 32.9(482.0-614.0)$ & $519.0 \pm 36.8(420.0-578.0)$ & $0.27^{\mathrm{a}}$ \\
Baseline MD (dB) & $10.7 \pm 3.1(5-20)$ & $10.0 \pm 3.8(6-24)$ & $0.07^{\mathrm{a}}$ \\
Baseline PSD (dB) & $-5.4 \pm 5.0(-23.7$ to 0.8$)$ & $-7.1 \pm 6.2(-23.3$ to 0.4$)$ & $0.26^{\mathrm{a}}$ \\
Baseline VFI (\%) & $8.2 \pm 5.1(1.6-16.8)$ & $8.0 \pm 4.8(1.6-18.0)$ & $0.93^{\mathrm{a}}$ \\
Progression rates of VFI by GPA (\%/year) & $85.5 \pm 14.8(31-99)$ & $79.1 \pm 20.2(22-100)$ & $0.15^{\mathrm{a}}$ \\
\hline
\end{tabular}

Abbreviations: CCT, central corneal thickness; GPA, glaucoma progression analysis; IOP, intraocular pressure; MD, mean deviation; PSD, pattern SD; RNFL, retinal nerve fiber layer; VFI, visual field index.

Statistically significant $(P<0.05)$ for mean difference between two groups. ${ }^{\text {at }}$-test, independent sample two-tailed $t$-test. ${ }^{b}$ Pearson's $\chi^{2}$-test. 

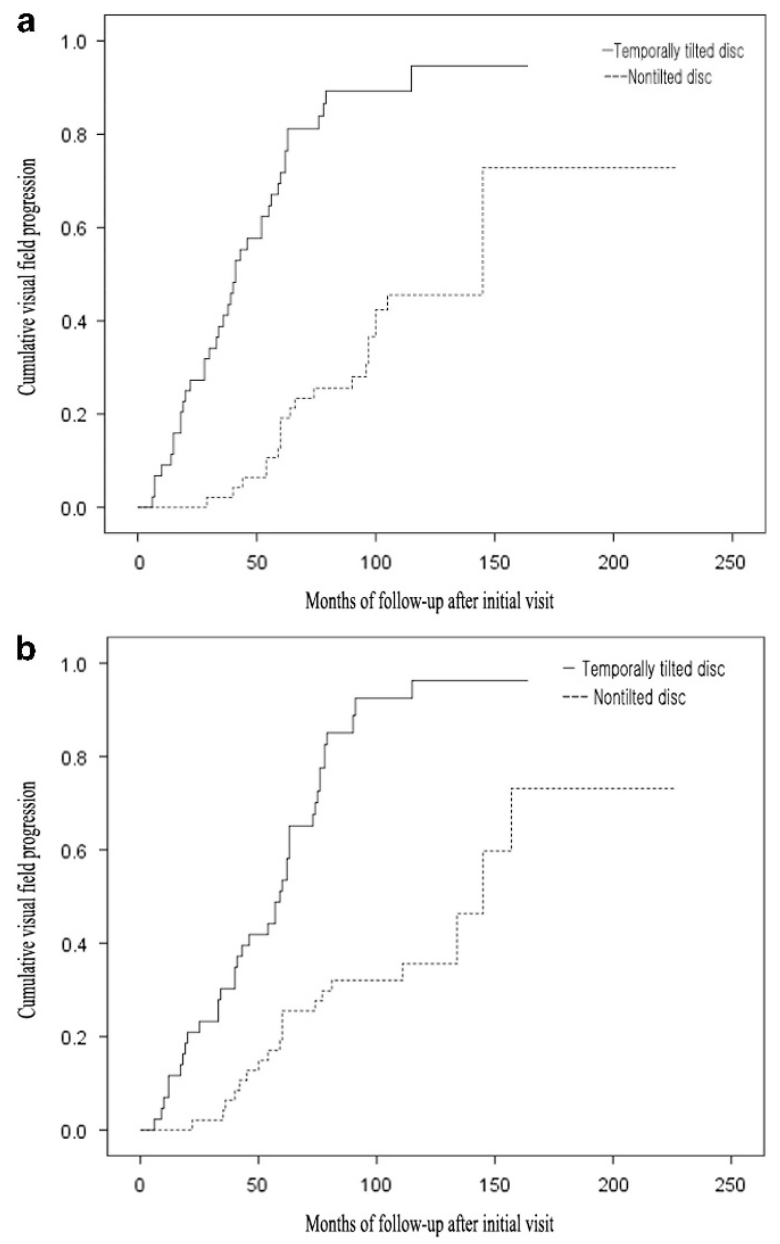

Figure 1 Kaplan-Meier survival plots of probabilities of visual field progression. The visual field progression was defined using two methods. Modified Anderson-Hodapp criteria (a) and Glaucoma Progression Analysis (GPA) software (b). According to the modified Anderson-Hodapp criteria, the progression rates of the two groups at 60 months were $19 \%$ in group 1 and $72 \%$ in the group $2\left(P<0.0001, \chi^{2}\right.$-test). According to the GPA, the progression rates 60 months after initial visit were $25 \%$ in group 1 and $53 \%$ in group 2 , respectively $\left(P<0.0001, \chi^{2}\right.$-test; temporally tilted disc: group 1, nontilted disc: group 2$)$.

non-progressors were 27 (Table 2). There were no statistically significant differences in age, gender, followup period, SE, number of topical medications, baseline IOP, baseline mean RNFL thickness, or baseline mean CCT. Also, the perimetric parameters, such as number of VF tests, baseline MD, baseline PSD, and baseline VFI were also not statistically different from each other.

We also evaluated the relationship between the IT and the MD value of the non-progressors in the temporally tilted disc. Mean of the IT in three eyes $(0.6<\mathrm{IT} \leq 0.7)$ was $0.637 \pm 0.025$ and the mean of the MD was $-12.920 \pm 8.523$. The mean of the IT in the remaining 24 eyes $(0.7<\mathrm{IT} \leq 0.8)$ was $0.766 \pm 0.098$ and the mean of the MD was $-5.219 \pm 4.127$.

\section{Multivariate analysis}

Risk factors of VF progression in temporally tilted disc in NTG patients We performed multivariate analysis, to find out the risk factors associated with the VF progression by the modified Anderson-Hodapp criteria in the temporally tilted disc. Other covariates included age, sex, number of topical medications, baseline mean RNFL thickness, baseline mean CCT, and baseline MD, This analysis was adjusted for the covariates, with multiple cox regression (Table 3). The significant risk factors for the progression were number of topical medications $(\mathrm{HR}=3.07 ; 95 \% \mathrm{CI}$, $1.08-8.76 ; P=0.04)$, RNFL defect type $(\mathrm{HR}=3.08 ; 95 \% \mathrm{CI}$, $1.17-8.14 ; P=0.02)$, and baseline $\mathrm{MD}(\mathrm{HR}=1.82 ; 95 \% \mathrm{CI}$, 1.05-3.17; $P=0.03)$. One unit increase of topical medications, RNFL defect type, and baseline MD increased the likelihood of the progression by factors of $3.07,3.08$, and 1.82 , respectively. The RNFL defect type was the most significant risk factor for the progression. Otherwise, increment of age and baseline mean RNFL thickness were associated with a decreased likelihood of progression, by factors of 0.94 (95\% CI, 0.89-0.99; $P=0.03$ ) and 0.94 (95\% CI, 0.89-0.99; $P=0.03$ ), respectively.

RNFL defect types of the progressors consisted of 12 eyes $(60 \%)$ with simultaneous superior and inferior RNFL defect, 5 eyes (25\%) with inferior RNFL defect, and 3 eyes $(15 \%)$ with superior RNFL defect. The simultaneous superior and inferior RNFL defect type was the most common. However, RNFL defect types of nonprogressors consisted of 18 eyes $(66.7 \%)$ with inferior RNFL defect, 5 eyes (18.5\%) with superior RNFL defect, and 4 eyes $(14.8 \%)$ with simultaneous superior and inferior RNFL defect. The inferior RNFL defect type accounted for the most part of the RNFL defect. The analysis of RNFL defect types showed statistically significant results $\left(P=0.04, \chi^{2}\right.$-test $)$.

\section{Discussion}

We investigated the long-term VF progression in the temporally tilted disc and the nontilted disc in NTG patients. In some studies, overlapping features with myopic disc and tilted disc explained the variation in prevalence, ${ }^{21,22}$ so we excluded eyes with myopia $>8$ D. ${ }^{14}$ In addition, patients who had systemic causes of a localized RNFL defect, such as arterial hypertension and diabetic mellitus, were not included. ${ }^{23,24}$ As mentioned earlier, we supposed that myopic tilted disc might not have VF progression. In a retrospective study by Doshi et al, ${ }^{9} 16$ glaucoma suspects or primary open angle glaucoma patients had stable ocular findings, including VF defects, for up to 7 years. In our study, 20 (43\%) of 47 patients in the temporally tilted disc had VF progression by the modified Anderson-Hodapp criteria, and 39 (87\%) 
Table 2 Comparison of the clinical demographics between progressors and non-progressors in temporally tilted disc

\begin{tabular}{|c|c|c|c|}
\hline Characteristics & Progressors $(\mathrm{n}=20)$ mean $\pm S D$ & Non-progressors $(\mathrm{n}=27)$ mean $\pm S D$ & P-value ${ }^{\mathrm{a}}$ \\
\hline Age (years) & $53 \pm 8.9(31-88)$ & $52 \pm 11.8(32-69)$ & 0.73 \\
\hline Gender male/female & $12 / 8$ & $18 / 9$ & 0.50 \\
\hline Follow-up period (months) & $101.1 \pm 28.9$ & $118.7 \pm 35.3$ & 0.07 \\
\hline Spherical equivalent (D) & $-4.8 \pm 1.5(-7$ to -3$)$ & $-4.9 \pm 1.2(-7$ to -0.2$)$ & 0.64 \\
\hline Number of topical medications & $1.3 \pm 0.5(1-2)$ & $1.5 \pm 0.5(1-3)$ & 0.08 \\
\hline Baseline IOP (mm Hg) & $16.0 \pm 2.8(13-20)$ & $16.8 \pm 2.5(10-21)$ & 0.35 \\
\hline Mean IOP (mm Hg) & $15.9 \pm 1.9(13.4-19.0)$ & $15.0 \pm 1.8(12.0-18.2)$ & 0.10 \\
\hline Peak IOP (mm Hg) & $19.0 \pm 2.3(16-21)$ & $18.0 \pm 2.6(14-21)$ & 0.24 \\
\hline Baseline mean RNFL thickness $(\mu \mathrm{m})$ & $81.4 \pm 13.8(58.4-106.1)$ & $75.3 \pm 15.2(48.68-104.58)$ & 0.16 \\
\hline Baseline mean CCT $(\mu \mathrm{m})$ & $532.1 \pm 35.5(482-588)$ & $527.4 \pm 29.3(484-614)$ & 0.64 \\
\hline Number of visual field tests & $10.5 \pm 3.4(8-16)$ & $11.1 \pm 2.4(5-20)$ & 0.52 \\
\hline Baseline MD (dB) & $-6.0 \pm 5.5(-11.4$ to -1.1$)$ & $-4.6 \pm 4.2(-23.74$ to -0.1$)$ & 0.34 \\
\hline Baseline PSD (dB) & $8.4 \pm 5.6(2.81-16.6)$ & $7.4 \pm 4.2(1.63-15.8)$ & 0.47 \\
\hline Baseline VFI (\%) & $84.4 \pm 15.5(56-99)$ & $87.1 \pm 13.9(31-99)$ & 0.54 \\
\hline
\end{tabular}

Abbreviations: CCT, central corneal thickness; IOP, intraocular pressure; MD, mean deviation; PSD, pattern SD; RNFL, retinal nerve fiber layer; VFI, visual field index. ${ }^{a}$ Mann-Whitney $U$-test.

Table 3 Multivariate risk factors associated with visual field progression by modified Anderson-Hodapp criteria in temporally tilted disc in normal tension glaucoma

\begin{tabular}{lccc}
\hline Variables & Hazard ratio & $95 \%$ CI & P-value \\
\hline Age & 0.94 & $0.89-0.99$ & 0.03 \\
Gender & 0.89 & $0.27-2.88$ & 0.84 \\
Number of topical medications & 3.07 & $1.08-8.76$ & 0.04 \\
Baseline IOP & 0.13 & $0.50-2.62$ & 0.40 \\
Mean IOP & 0.45 & $0.18-2.01$ & 0.20 \\
Peak IOP & 0.26 & $0.60-2.35$ & 0.42 \\
Baseline mean RNFL thickness & 0.94 & $0.89-0.99$ & 0.03 \\
Baseline mean CCT & 1.00 & $0.98-1.02$ & 0.78 \\
RNFL defect type & 3.08 & $1.17-8.14$ & 0.02 \\
Baseline MD & 1.82 & $1.05-3.17$ & 0.03 \\
\hline
\end{tabular}

Abbreviations: CCT, central corneal thickness; CI, confidence interval; $\mathrm{IOP}$, intraocular pressure; $\mathrm{MD}$, mean deviation; RNFL, retinal nerve fiber layer. ${ }^{a}$ Multiple Cox regression.

of 44 patients in the nontilted disc in NTG patients had VF progression.

One possible explanation for this is that progressive myopia leads to progressive increase in axial length, progressive thinning of the posterior choroid and sensory retina, and progressive tilting of the optic disc, make it susceptible to axonal loss. ${ }^{9}$ The tilted disc gives rise to asymmetric increase in strain on nerve fibers, therefore susceptible axons are subsequently lost. However, if progression of myopia stopped at a certain age the VF defect will not progress further.

The second explanation is that the weakest inferotemporal LC has the least connective tissue and the largest pores, ${ }^{12,25}$ therefore, myopic tilting of the optic disc causes more distortion of the inferotemporal pore of the LC (Figure 2). Thus, the axons passing through the inferotemporal pore may be damaged easily, and result in glaucomatous RNFL defect that cannot be distinguished from those caused by glaucoma. However, if myopia does not proceed further, RNFL will not be damaged any more.

The progression rate of the VFI in the nontilted disc was faster than the temporally tilted disc. In addition, the cumulative survival rates were significantly higher in the temporally tilted disc, than the nontilted disc. The progression rates of the progressors between the two groups were not statistically different, as well as the progressors over 6 D in group 1 vs the progressors in group 2. Likewise, there were no significant differences in baseline MD and baseline PSD between the groups 1 and 2 . These findings were consistent with previous study by Sohn et $a l^{5}$ that evaluated the influence of the extent of myopia on the progression rate of NTG. They divided myopic patients into three groups: mild myopia ( -0.76 to $-2.99 \mathrm{D})$, moderate myopia ( -3 to $-5.99 \mathrm{D})$, and severe myopia ( $-6 \mathrm{D}$ or less). The results of their study also showed that there was no significant difference between the nonmyopia group and each of the myopia groups in MD, and PSD. In other words, myopia did not influence the progression rate of NTG after treatment, as in our study.

We evaluated the relationship between the IT and the MD value of the non-progressors, in the temporally tilted disc. The more tilted disc seemed to have a higher MD value. Accordingly, the more tilted disc may cause more RNFL defect. We define the tilted disc as being when IT was $\leq 0.8$ and assessed the validity of IT using a value $<0.85$, based on How et al. ${ }^{26}$ Its sensitivity was found to be $56.7 \%$ and the specificity was $94.0 \%$, using stereoscopic photographs.

We have shown that several factors were significantly associated with the VF progression by the modified Anderson-Hodapp criteria in the temporally tilted disc. The multivariate analysis demonstrated a significant association between young age and the VF progression (Table 3). 

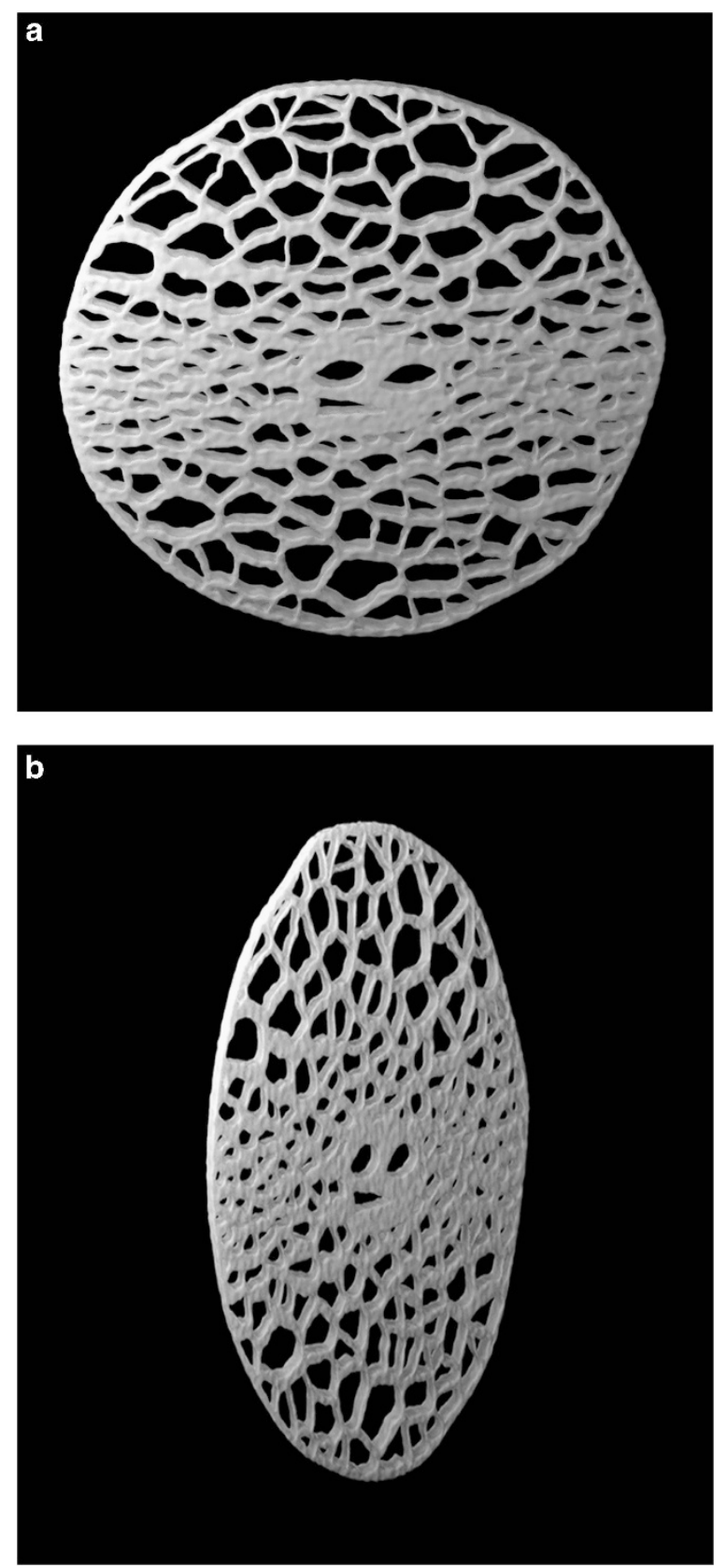

Figure 2 Three-dimensional schematic image of normal LC (a) and temporally tilted LC with vertical section (b).

However, Sakata et $a l^{27}$ and Drance et $a l^{28}$ found no significant correlation between the age and VF progression in NTG. Number of topical medications, baseline thin mean RNFL thickness, and high baseline MD were also associated with VF progression. Similary, Lyu et al ${ }^{29}$ reported that the number of topical medications was strongly associated with the VF progression. However, the number of ocular drugs can be interpreted as a secondary result of the rapid VF defect. Also, thin RNFL and high baseline MD were susceptible to VF damage. ${ }^{28,30}$ We were interested to find that the spontaneous superior and inferior RNFL defect may become a predictor of the VF progression. Disc tilt usually does not emerge at the superior region; so, existence of the spontaenous superior and inferior RNFL defect imply that care should be taken.

As IOP is the only controllable risk factor for progression, the doctor will prescribe additional topical medication to patients with suspected persistent VF progression to suppress progression. In other words, multiple topical medications can be interpreted as a secondary result, in accordance with fast VF progression.

Our study has several limitations. Topical medication from the time of diagnosis of the temporally tilted disc might have inadvertently affected VF defects, possibly slowing the VF progression. However, the patients were prescribed the same eyedrops and had a similar range of IOP, so it may be reasonable to assume that the impact from topical medication, if any, would be minimal. The limitation of this study also include it being retrospective, with a relatively small number of patients, and having variable follow-up. However, our study has the longest follow-up of the temporally tilted disc in NTG patients investigating the VF progression rates and looking into the associated risk factors, and provides reasonably good insight into starting the medical treatment. In addition, receiving treatment based on consistent principles by a doctor and performing multiple VF tests during the long follow-up period were the advantages of our study.

In conclusion, the cumulative survival rate and progression rates of the temporally tilted disc in NTG patients were higher than of the nontilted disc during longterm follow-up. The progression rates between the progressors in the temporally tilted disc and those in the nontilted disc were not different. Twenty (57\%) patients with the temporally tilted disc in NTG did not show progression. Among them, the more non-progressed tilted disc showed the more baseline VF defects. Therefore, even severely defected VF has not shown any more progress in the temporally tiled disc in NTG patients, so they will not need aggressive treatment. Caution is warranted in the treatment of the temporally tilted disc in NTG.

\section{Summary}

What was known before

- The tilted disc merely caused by myopic change might not have progression, even if RNFL defects exist.

\section{What this study adds}

- We compared the long-term VF progression between temporally tilted disc and nontilted disc in NTG. In addition, we analyzed the risk factors associated with VF progression of the temporally tilted disc in NTG, and evaluated the differences of progression rate in progressors, between temporally tilted disc and nontilted disc, in NTG. 


\section{Conflict of interest}

The authors declare no conflict of interest

\section{Author contribution}

Y-JC and CK designed the study; Y-JC, YK, JCH and CK conducted the study; Y-JC, YK and CK were involved in data collection and management, analysis and interpretation of data; Y-JC, YK and CK prepared, reviewed, and approved the manuscript. The present study was approved by the Institutional Review Board of Samsung Medical Center, Sungkyunkwan University School of Medicine, Seoul, Korea. An exemption from informed consent for research was granted, because this was a retrospective research study.

\section{References}

1 Tezel G, Trinkaus K, Wax MB. Alterations in the morphology of lamina cribrosa pores in glaucomatous eyes. $\mathrm{Br} J$ Ophthalmol 2004; 88: 251-256.

2 Quigley HA, Hohman RM, Addicks EM, Massof RW, Green WR. Morphologic changes in the lamina cribrosa correlated with neural loss in open-angle glaucoma. Am J Ophthalmol 1983; 95: 673-691.

3 Kim TW, Kagemann L, Girard MJ, Strouthidis NG, Sung KR, Leung CK et al. Imaging of the lamina cribrosa in glaucoma: perspectives of pathogenesis and clinical applications. Curr Eye Res 2013; 38: 903-909.

4 Wong TY, Klein BE, Klein R, Knudtson M, Lee KE. Refractive errors, intraocular pressure, and glaucoma in a white population. Ophthalmology 2003; 110: 211-217.

5 Sohn SW, Song JS, Kee C. Influence of the extent of myopia on the progression of normal-tension glaucoma. Am J Ophthalmol 2010; 149: 831-838.

6 Mitchell P, Hourihan F, Sandbach J, Wang JJ. The relationship between glaucoma and myopia: the Blue Mountains Eye Study. Ophthalmology 1999; 106: 2010-2015.

7 Quigley HA, Anderson DR. Distribution of axonal transport blockade by acute intraocular pressure elevation in the primate optic nerve head. Invest Ophthalmol Vis Sci 1977; 16: 640-644.

8 Radius RL, Anderson DR. Rapid axonal transport in primate optic nerve. Distribution of pressure-induced interruption. Arch Ophthalmol 1981; 99: 650-654.

9 Doshi A, Kreidl KO, Lombardi L, Sakamoto DK, Singh K. Nonprogressive glaucomatous cupping and visual field abnormalities in young Chinese males. Ophthalmology 2007; 114: 472-479.

10 Park HY, Lee K, Park CK. Optic disc torsion direction predicts the location of glaucomatous damage in normaltension glaucoma patients with myopia. Ophthalmology 2012; 119: $1844-1851$.

11 Shields MB. Textbook of Glaucoma, 6th ed. Williams \& Wilkins: Baltimore, 1998; pp 178-179.

12 Cho HK, Kee C. Comparison of the progression rates of the superior, inferior, and both hemifield defects in normal- tension glaucoma patients. Am J Ophthalmol 2012; 154: 958-968 e951.

13 Tay E, Seah SK, Chan SP, Lim AT, Chew SJ, Foster PJ et al. Optic disk ovality as an index of tilt and its relationship to myopia and perimetry. Am J Ophthalmol 2005; 139: 247-252.

14 You QS, Xu L, Jonas JB. Tilted optic discs: The Beijing Eye Study. Eye (Lond) 2008; 22: 728-729.

15 Khan KA, Dawson K, Mankowska A, Cufflin MP, Mallen EA. The time course of blur adaptation in emmetropes and myopes. Ophthalmic Physiol Opt 2013; 33: 305-310.

16 Kim JW, Chen PP. Central corneal pachymetry and visual field progression in patients with open-angle glaucoma. Ophthalmology 2004; 111: 2126-2132.

17 Chen PP. Correlation of visual field progression between eyes in patients with open-angle glaucoma. Ophthalmology 2002; 109: 2093-2099.

18 Rao HL, Kumbar T, Kumar AU, Babu JG, Senthil S, Garudadri CS. Agreement between event-based and trend-based glaucoma progression analyses. Eye (Lond) 2013; 27: 803-808.

19 Rao HL, Kumar AU, Babu JG, Senthil S, Garudadri CS. Relationship between severity of visual field loss at presentation and rate of visual field progression in glaucoma. Ophthalmology 2011; 118: 249-253.

20 Bengtsson B, Heijl A. A visual field index for calculation of glaucoma rate of progression. Am J Ophthalmol 2008; 145: 343-353.

21 Riise D. The nasal fundus ectasia. Acta Ophthalmol Suppl 1975; 126: 3-108.

22 Giuffre G. Tilted discs and central retinal vein occlusion. Graefes Arch Clin Exp Ophthalmol 1993; 231: 41-42.

23 Lee J, Kim J, Kee C. Characteristics of patients with a localized retinal nerve fiber layer defect and normal optic disc appearance. Eye (Lond) 2012; 26: 1473-1478.

24 Hayreh SS, Jonas JB. Appearance of the optic disk and retinal nerve fiber layer in atherosclerosis and arterial hypertension: an experimental study in rhesus monkeys. Am J Ophthalmol 2000; 130: 91-96.

25 Dandona L, Quigley HA, Brown AE, Enger C. Quantitative regional structure of the normal human lamina cribrosa. A racial comparison. Arch Ophthalmol 1990; 108: 393-398.

26 How AC, Tan GS, Chan YH, Wong TT, Seah SK, Foster PJ et al. Population prevalence of tilted and torted optic discs among an adult Chinese population in Singapore: the Tanjong Pagar Study. Arch Ophthalmol 2009; 127: 894-899.

27 Sakata R, Aihara M, Murata H, Mayama C, Tomidokoro A, Iwase A et al. Contributing factors for progression of visual field loss in normal-tension glaucoma patients with medical treatment. J Glaucoma 2013; 22: 250-254.

28 Drance S, Anderson DR, Schulzer M. Risk factors for progression of visual field abnormalities in normal-tension glaucoma. Am J Ophthalmol 2001; 131: 699-708.

29 Lyu IJ, Lee JM, Kee C. Risk factors for rapid visual field progression in normal-tension glaucoma. J Korean Ophthalmol Soc 2012; 53: 996-1001.

30 Tenkumo K, Hirooka K, Baba T, Nitta E, Sato S, Shiraga F. Evaluation of relationship between retinal nerve fiber layer thickness progression and visual field progression in patients with glaucoma. Jpn J Ophthalmol 2013; 57: 451-456. 